\title{
Retraction Note: The amine-catalysed Suzuki-Miyaura-type coupling of aryl halides and arylboronic acids
}

Lei Xu (D), Fu-Yue Liu, Qi Zhang, Wei-Jun Chang, Zhong-Lin Liu, Ying Lv, Hai-Zhu Yu (D), Jun Xu (D), Jian-Jun Dai (iD and Hua-Jian Xu $\mathbb{D}$

Retraction to: Nature Catalysis, https://doi.org/10.1038/s41929-020-00564-z, published online 18 January 2021.

We wish to retract this article as we can no longer claim that the observed reactivity for the Suzuki-Miyaura-type coupling of aryl halides and arylboronic acids is attributed to the amine organocatalyst. Efforts from different laboratories-published in the form of Matters Arising articles ${ }^{1-3}$ - have pointed to the fact that the reaction is catalyzed by residual palladium impurity contained in the amine. We have performed a series of validation experiments confirming that during the synthesis of the amine, stable palladium complexes are formed, which could not be completely removed by column chromatography. Moreover, the ICP-MS method we used could not completely digest the residual palladium. Such factors led us to misjudge the nature of the catalyst.

We are very grateful to the authors of the Matters Arising articles for raising these issues. We sincerely apologize to the scientific community for any confusion arising from our publication. All authors agree with this retraction.

\section{References}

1. Novák, Z. et al. Nat. Catal. https://doi.org/10.1038/s41929-021-00709-8 (2021).

2. Avanthay, M. et al. Nat. Catal. https://doi.org/10.1038/s41929-021-00710-1 (2021).

3. Vinod, J.K. Nat. Catal. https://doi.org/10.1038/s41929-021-00711-0 (2021).

Published online: 8 December 2021

https://doi.org/10.1038/s41929-021-00726-7

(c) The Author(s), under exclusive licence to Springer Nature Limited 2021 